\title{
Does Everything Get Slower with Age? Pharmacokinetics in the Elderly
}

The pharmacokinetics (PK) of a drug determine its systemic exposure and thereby affect its safety and efficacy. The multitude of physiological changes occurring with aging could impact drug absorption, distribution, metabolism or excretion, the key processes how the body is dealing with drugs. Another important, PK-related topic is the risk for relevant drug-drug interactions considering the number of drugs taken concomitantly by elderly patients. At the same time there are examples for relevant differences in the magnitude of drug effects, i.e. the pharmacodynamics of a drug in an aged vs. young population. These could be caused by differences in the number of receptors in the target organ as well as the response to receptor occupation, or by progressive reduction in homeostatic mechanisms, and could affect the efficacy as well as the safety [1]. Such age-related changes in the pharmacodynamics of a drug shall, however, not be addressed in this article despite their proven clinical relevance.

When discussing age-related changes in the PK of drugs, and in particular their consequences for dosing recommendations in aged patients, one needs to keep in mind that aging is a gradual, complex and progressive process and that there are no established biological age markers. While there is societal consensus to define elderly as individuals aged 65 years or older [1], dividing elderly in three age groups, such as 65-75, 76-85, and > 85 might help in better describing the aging processes [2].

\section{Absorption}

As most drugs are administered perorally, age-related changes of the gastrointestinal tract could be expected to be a major source of PK differences with age. Such age-related physiological changes include, e.g. decreased absorption surface and gastrointestinal motility, delayed gastric emptying, increased gastric $\mathrm{pH}$. The gastrointestinal absorption of most drugs, in particular when permeating by passive diffusion, remains, however, unchanged $[1,2]$. This can be different for drugs affected by an extensive first-pass metabolism show- ing a marked increase in bioavailability in some cases $[3,4]$.

Other routes of administration, e.g. transdermal, subcutaneous or intramuscular, might be affected by reduced tissue blood perfusion resulting in lower rate of drug absorption [1], although e.g. for the skin such changes were not considered to be clinically relevant [5]. Age-related changes in lung function (e.g. increased lung rigidity resulting in narrower respiratory capacity) or the eye (e.g. reduced tear film) can affect drug absorption via these routes [5].

\section{Distribution}

Changes in the body composition with advancing age, like increase in body fat and decrease in plasma volume and body water, can lead to an increase of the apparent volume of distribution of lipophilic drugs and its decrease for hydrophilic drugs [6] with potential consequences on the elimination half-life. Little clinical relevance is attributed to age-related changes in protein binding [2].

\section{Metabolism}

Despite the consensus on a marked agerelated reduction in liver mass and hepatic blood flow, the effect of age on hepatic drug metabolism continues to be controversial [1]. There is evidence for a decrease in cytochrome P450 (CYP) oxidases with age resulting in decreased clearance [7, 8]. At the same time, there are numerous examples of drugs subject to CYP-mediated metabolism, but with unchanged clearance in the elderly [1]. These latter findings are in line with several in vitro investigations on hepatic enzyme content and activity investigated in hepatocytes and liver microsomes of different age groups [2]. There is consensus in the community that the activity of phase 2 metabolic enzymes is preserved in the elderly as well as the ability for and extent of enzyme induction $[2,6]$.

\section{Excretion}

The apparently most prominent change affecting the PK of predominantly renally excreted drugs is the age-related decline in renal function. Glomerular filtration rate (GFR) declines by $25-50 \%$ between the ages of 20 and 90 [1]. About $1 / 3$ of the population is, however, not affected by these changes [2]. Studies suggest that the decline in renal function is more caused by confounding factors like hypertension or chronic heart disease than by age per se [9]. The Cockroft-Gault, MDRD or CKD-EPI equations allow easy calculation of renal creatinine clearance as an indicator of GFR based on serum creatinine concentrations, body weight and age and can be used for calculating dose adjustment.

\section{Drug-drug interactions}

The number of drugs taken concomitantly increases with the appearance of multimorbidities in the elderly. This increases the risk for drug-drug interactions (DDI) and resulting adverse drug reactions relevantly. A study in 1600 patients above the age of 65 in 6 European countries revealed a concomitant intake of 7 drugs on average in this population, thereof at least one combination possibly leading to a DDI in $46 \%$ of the patients [10].

\section{Conclusions}

Despite several physiological changes occurring with aging, their sole impact on the PK of most drugs is limited. Disease-related changes, in frail patients in particular, as well as polypharmacy in the aged appear to have the more relevant effect. With that and the age-related changes on the pharmacodynamic side, elderly remain a vulnerable patient population affected by a 2-3-fold higher rate of adverse drug reactions than adult patients below the age of 30 years [1]. In the absence of general rules, the individual product label needs to be consulted in order to mitigate the risk for adverse reactions in the elderly. 


\section{Conflict of Interest}

Joachim Höchel is employee of Bayer AG and has no other conflict of interest to declare.

\section{Author}

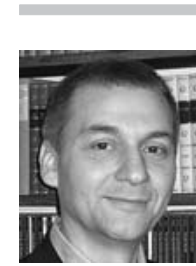

\section{Joachim Höchel}

Clinical Pharmacology Gynecological Therapies and Specialized Therapeutics, Pharmaceutics Research and Development, Bayer AG, Germany

\section{Correspondence}

\section{Dr. Joachim Höchel}

Bayer AG

13342 Berlin

Germany

joachim.hoechel@bayer.com

\section{References}

[1] Turnheim K. When drug therapy gets old: pharmacokinetics and pharmacodynamics in the elderly. Exp Gerontol 2003; 38: 843853

[2] Klotz U. Pharmacokinetics and drug metabolism in the elderly. Drug Metab Rev 2009; 41: 67-76

[3] Nation RL, Vine J, Triggs EJ et al. Plasma level of chlormethiazole and two metabolites after oral administration to young and aged human subjects. Eur J Clin Pharmacol 1977; 12: $137-145$

[4] Castleden CM, George CF. The effect of ageing on the hepatic clearance of propranolol. Br J Clin Pharmacol 1979; 7: 49-54

[5] Perrie Y, Badhan RKS, Kirby D] et al. The impact of ageing on the barriers to drug delivery. J Control Release 2012; 161: 389-398
[6] Pea F. Pharmacokinetics and drug metabolism of antibiotics in the elderly. Expert Opin Drug Metab Toxicol 2018; 14: 1087-1100

[7] Sotaniemi EA, Arranto AJ, Pelkonen $O$ et al. Age and cytochrome P450-linked drug metabolism in humans: An analysis of 226 subjects with equal histopathologic conditions. Clin Pharmacol Ther 1997; 61: 331-339

[8] Cotreau MM, von Moltke LL, Greenblatt D]. The influence of age and sex on the clearance of cytochrome P450 3A substrates. Clin Pharmacokinet 2005; 44: 33-60

[9] Fliser D, Franek E, Joest $M$ et al. Renal function in the elderly: impact of hypertension and cardiac function. Kidney Int 1997; 51: 1196-1204

[10] Björkman IK, Fastbom J, Schmidt IK et al. Drug-drug interactions in the elderly. Ann Pharmacother 2002; 36: 1675-1681 